\title{
Detection of ball grid array solder joints based on adaptive template matching
}

Xueli Hao, Wei Li*, Zhaoyun Sun, Shaojun Zhu, Shuai Yan, Zhao Zhao

School of Information Engineering, Chang'an University, Xi'an, China

Corresponding Author Email: houqing841128@163.com

https://doi.org/10.18280/ijht.360125

Received: 20 August 2017

Accepted: 1 October 2017

Keywords:

adaptive template matching, automatic thresholding, ball grid array (bga), edge direction vector, image pyramid

\begin{abstract}
This paper aims to achieve the accurate detection of ball grid array (BGA) solder joints. To this end, the author presented an adaptive template matching method for BGA solder joints based on shape detection. First, the region of interest (ROI) was selected from the X-ray image of the printed circuit board (PCB). Then, an edge template was generated through ROI extraction and threshold segmentation, and the direction vector $\mathrm{f}$ the edge template was taken as the prior knowledge. After that, the global traversal search was performed on the image pyramid in the top-down manner, aiming to obtain the potential matching targets. Finally, the edges were adjusted by the least squares method to yield the optimal matching results. The proposed method was proved robust, rapid and accurate through an experiment. The research findings shed new light on the BGA solder joint detection and extraction in various conditions.
\end{abstract}

\section{INTRODUCTION}

The microelectronics integration is featured by the reduction of chip size and the increase of $\mathrm{I} / \mathrm{O}$ port pins. Currently, the pins are made in various shapes. Among them, the spherical ball grid array (BGA) solder joint stands out for the following reasons: numerous I/O points, no pin distortion, low self-inductance and mutual inductance, and favorable frequency and coplanarity. Thanks to these advantages, the spherical BGA solder joints, integrated with the popular surface-mount technology (SMT), has been extensively adopted as a new form of chip package [1-4]. Against this backdrop, it is very important to ensure the quality of BGA solder joints.

Over the years, many image segmentation methods have been developed to extract BGA solder joints from the X-ray images of printed circuit board (PCB), such as threshold segmentation and edge extraction, to name but a few [5-12]. The X-ray technique is adopted because it is impossible to find the defects in visible light. After all, the BGA solder joints are hidden beneath the integrated circuit package. Below is a brief review of relevant studies.

Through SAC nanomechanical testing, Hasnine et al. [13] explored the aging phenomenon of the lead-free solder joints extracted from plastic BGA assemblies. Said et al. [14] proposed an accurate and efficient non-wet detection method, which involves region of interest (ROI)segmentation, feature extraction, reference-free classification and automatic mapping. Pierce [15] developed a preliminary automated inspection system and combined it with artificial neural network (ANN) to inspect the solder joints in an X-ray image. Park [16] extracted 15 features from four frames in solder joint images, divided solder joint defects into 7 categories based on these features and provided two kinds of inspection results depending on their uses. Koh et al. [17] put forward a time and labour saving statistical learning approach based on part recognition. Lahari [18] created an image processing and

computer vision technique, that can detect every joint in the X-ray image on BGA chip and flag the suspect joints for further evaluation and rework. Deokhwa [19] presented a multi-screen deflectometer to increase the measurable slope angle of solder joints but failed to achieve a good detection accuracy under complicated background.

To sum up, the above studies cannot meet the demand of actual industrial detection, despite their deliberations on background noises and various defects. To solve the problem, the robust, adaptive templating matching method of automatic thresholding was proposed to detect the BGA solder joints rapidly and efficiently.

\section{X-RAY IMAGING OF BGA}

As mentioned before, X-ray imaging is often adopted for BGA solder joints due to the overlapping between BGA packages and other PCB components. The identification and extraction of BGA solder joints are complicated by the following factors: the greyscale difference between components and the surroundings, the interconnection among several grey areas, the various noises in the background, and the interference from the components around the joint (Figure 1).

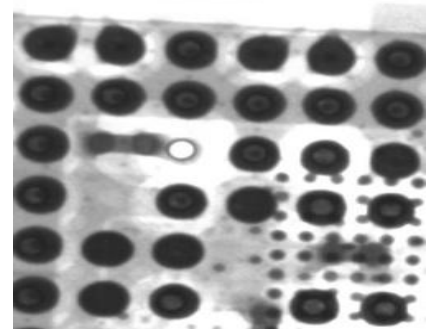

(a)

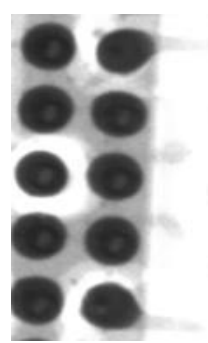

(b)

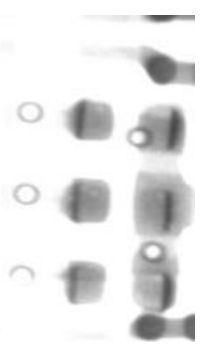




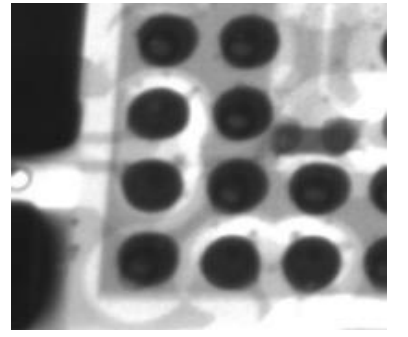

(c)

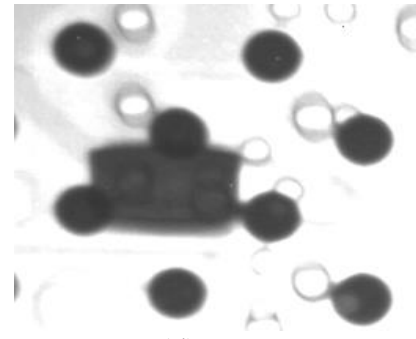

(d)
Figure 1. Influencing factors of BGA solder joint identification and extraction

In Figure (a), the background of BGA solder joints contain too many noises, including welding plate and small solder joints. In Figure 1(b), the BGA solder joints are difficult to distinguish from the near-round welding plate and the surrounding devices. In Figure 1(c), the BGA solder joints share a similar greyscale with the peripheral area. In Figure 1(d), the BGA solder joints are contaminated by foreign substances. During the soldering of BGA packages, the molten solder tends to distribute in a spherical shape due to the surface tension. As shown in Figure 2, the solder at the centre of the sphere is thicker than that on the grey edge. The black central area has a lower greyscale than the edge.

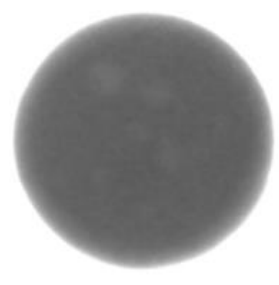

Figure 2. The spherical shape of BGA solder joints

In general, all BGA solder joints are near-circular on the edge. However, slight variation in shape occurs, as each BGA solder joint has its own degree of deformation. Coupled with the complex background, the shape difference makes it difficult to extract BGA solder joints with a global threshold. What is worse, the greyscale-based ridged template cannot identify solder joints accurately, facing the nonuniform greyscale and various deformations. In light of these, the author extracted the edge shapes of BGA solder joints and took them as the prior knowledge for characterizing the joints. Then, matching parameters were adjusted by the least squares method, based on the feature variation of direction vector at each edge point. The purpose is to achieve a desirable result on image registration and joint detection.

\section{ADAPTIVE TEMPLATE MATCHING}

\subsection{Template matching}

The template matching is either based on greyscale or features. The greyscale-based method measures the correlation between the template and the object by the similarity of image greyscale. This method has a low robustness because it is sensitive to the deformation, rotation and uneven illumination of the object. Sometimes, it is impossible to find a matching template by this method. The most popular greyscale-based template matching approach is zero normalized cross-correlation. Considering the inconsistent shape and nonuniform greyscale distribution, it is safe to say that the greyscale-based method does not apply to BGA solder joints in our research.

By contrast, the feature-based method describes the object with features like edge and contour and evaluates the correlation between the template and the object based on these features. Using the edge and contour features, the feature-based template matching can be adopted for BGA solder joints detection in our research. Similar to parameter estimation, the featured-based template matching obtains the coordinates of the object in the image through solving correlation functions. The specific steps are as follows:

The first step is to translate the template function $\mathrm{T}(\mathrm{x}, \mathrm{y})$ of the original image. In the original image, the block covered by the template was defined as subgraph $S^{i, j}$, where $(i, j)$ are the coordinates of the upper left corner of the subgraph in the original image. The similarity between template $\mathrm{T}(\mathrm{x}, \mathrm{y})$ and subgraph $S^{i, j}$ can be expressed as:

$\mathrm{R}(i, j)=\sum_{x=0}^{m-1} \sum_{y=0}^{n-1}\left[T(x, y)-S^{i, j}(x, y)\right]^{2}$

where $m \cdot n$ is the window size for solving the template function; $(\mathrm{x}, \mathrm{y})$ are the coordinates of each pixel in the original image. Then, Equation (1) can be expanded into:

$$
\begin{aligned}
& \mathrm{R}(i, j)=\sum_{x=0}^{m-1} \sum_{y=0}^{n-1}\left[S^{i, j}(x, y)\right]^{2}-\sum_{x=0}^{m-1} \sum_{y=0}^{n-1} S^{i, j}(x, y) * T(x, y) \\
& +\sum_{x=0}^{m-1} \sum_{y=0}^{n-1}[T(x, y)]^{2}
\end{aligned}
$$

where the first term is the energy of the subgraph, i.e. the block covered by the template in the original template; the second term is the relationship between the original image and the template; the third term is the energy of the template. The value of the second term is negatively correlated with $\mathrm{R}(\mathrm{i}, \mathrm{j})$. If the second term reaches its peak, it means the highest similarity appears between the current subgraph and the template, and the optimal matching area exists in the original image.

\subsection{Adaptive template matching}

\subsubsection{Image preprocessing}

The X-ray image acquired by industrial camera is a singlechannel greyscale image. In many cases, the image has a complex background and poor quality, making it hard to extract the exact BGA solder joints (Figure 3(a)). To ensure the accuracy of subsequent detection and application, it is necessary to enhance the quality of the original image. Therefore, the edges and other details of the original image should be highlighted and enhanced, so that the edge information can be extracted for template matching. Specifically, the $5 \times 7$ matrix below should be adopted as a linear filter to remove the low-frequency components from the original image, leaving only the high-frequency components. The filtered image is displayed in Figure 3(b) 


$\left[\begin{array}{ccccccc}1 & 1 & 1 & 1 & 1 & 1 & 1 \\ 1 & 1 & 1 & 1 & 1 & 1 & 1 \\ 1 & 1 & 1 & -35 & 1 & 1 & 1 \\ 1 & 1 & 1 & 1 & 1 & 1 & 1 \\ 1 & 1 & 1 & 1 & 1 & 1 & 1\end{array}\right]$

Next, the original image should be subtracted, and the zero-crossing point with the value of 128 will be returned. The cutoff frequency of the filter depends on the size of the filter matrix. The larger the matrix,the lower the frequency.

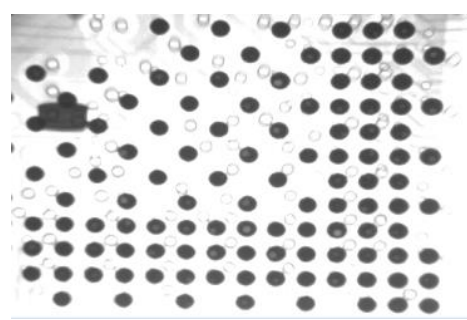

(a) Original image

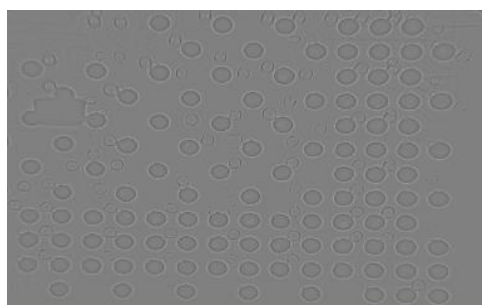

(b) Pre-processed image

Figure 3. Image preprocessing

\subsubsection{Similarity measure}

After preprocessing, the high-frequency components of the original image, namely, the target edges of BGA solder joints, are enhanced successfully, laying the basis for accurate recognition.

For any point on the template edge $p_{i}=$ $\left(x_{i}, y_{i}\right)^{T}(\mathrm{i}=1,2,3 \ldots \mathrm{n})$, calculate the edge of the corresponding direction vector $d_{i}=\left(u_{i}, v_{i}\right)^{T}(\mathrm{i}=1,2,3 \ldots \mathrm{n})$ using the Canny edge detector, an operator for image searching and matching. The direction vector of each edge point $(\mathrm{x}, \mathrm{y})$ is denoted ass $s_{x, y}=\left(r_{x, y}, c_{x, y}\right)^{T}$.

Let $M$ be the second-order standard rotation matrix. Perform affine transformation on the edge template and separate the corresponding transformation components. The edge points $p_{i}^{\prime}=M p_{i}$ and the direction vector $d_{i}^{\prime}=M d_{i}$ can be obtained after the transformation. Next, match the template with the original image at a certain point $q=(x, y)^{T}$, conduct dot product between the normalized direction vector of the points in the edge template and that of the corresponding points in the original image, and find the similarity measure $\mathrm{R}$ of point $\mathrm{q}$ in the original image through summation.

$$
\begin{aligned}
& R=\frac{1}{n} \sum_{i=1}^{n} \frac{\left(d_{i}^{\prime}\right)^{T} s_{p^{\prime}+q}}{\left\|\left(d_{i}\right)^{T}\right\| \cdot\left\|s_{p^{\prime}+q}\right\|} \\
& =\frac{1}{n} \sum_{i=1}^{n} \frac{x_{i}^{\prime} r_{x+x_{i}^{\prime} y+y_{i}^{\prime}}+y_{i}^{\prime} c_{x+x_{i}^{\prime} y+y_{i}^{\prime}}}{\sqrt{x_{i}^{2}+y_{i}^{2}} \sqrt{r_{x+x_{i}^{\prime} y+y_{i}^{\prime}}^{2}+c_{x+x_{i} y+y_{i}^{\prime}}^{2}}}
\end{aligned}
$$

If some of the features are missing in the edge template or original image, the direction vector will be less accurate because of the image noises. In this case, the mean direction vector of edge points will be affected, which will, in turn, influence the total similarity. To prevent the issue, the direction vector of isotropic pixel points should be set to zero, and only these pixels should be retained and expressed with normalized direction vectors. These measures ensure that the total similarity is immune from local noises (e.g. blocking of solder joint and complex background) and guarantee the antiinterference ability of the extraction of BGA solder joints. Overall, the total similarity measure can withstand illumination changes and uneven illumination, because the modulus value of direction vector depends on image brightness, and all direction vectors in the similarity measure should be normalized to 1 .

The similarity formula can be expressed as:

$R=\frac{1}{n} \sum_{i=1}^{n} \frac{\left|\left(d_{i}^{\prime}\right)^{T} s_{p^{\prime}+q}\right|}{\left\|\left(d_{i}\right)^{T}\right\| \cdot\left\|s_{p^{\prime}+q}\right\|}$

In the course of template matching, the local brightness contrast does not affect the similarity score. In some cases, however, the image contrast is completely reversed. To locate the object under reversed contrast, the similarity formula can be modified as:

$R=\left|\frac{1}{n} \sum_{i=1}^{n} \frac{\left(d_{i}^{\prime}\right)^{T} s_{p^{\prime}+q}}{\left\|\left(d_{i}\right)^{T}\right\| \cdot\left\|s_{p^{\prime}+q}\right\|}\right|$

The potential objects can be filtered and the matching speed can be improved by restricting the similarity score with the threshold $R_{m, n}$ :

$R_{j}=\frac{1}{n} \sum_{i=1}^{j}\left(d_{i}^{\prime}\right)^{T} s_{p^{\prime}+q}$

If $R_{j}<R_{m, n}-1+\lambda \mathrm{n}$, the similarity score is below the threshold $R_{m, n}$.In this case, the matching computation should stop immediately after handling the $\mathrm{j}$-th element. In this way, the computing load and matching time will be substantially reduced

\subsubsection{Match searching and position adjustment}

To ensure the accuracy of matching results on the subpixel level, the global traversal search should be performed first in the top layer of the image pyramid, and the similarity scores of all potential matching positions should be acquired by similarity measure calculation. Then, the potential matching positions and their transformation parameters will be returned. In light of the parameters, the BGA edge template should be subjected to an adaptive transformation. The local maximum similarity score of the potential matching positions must be greater than the threshold Rm,n. Otherwise, the computation should be terminated. These measures can greatly improve the computing efficiency. Thus, the matching and positioning should be performed layer by layer in a top-down manner. 
For the sub-pixel-level matching accuracy, the matching parameters should be adjusted by the least squares method. Let $q_{i}=\left(x_{i}^{\prime}, y_{i}^{\prime}\right)^{T}$ be the potential matching points between the image and the template. Since the edge point vectors in the template are vertical to the target edge, the tangent line of the edge passing through the potential matching points in the templates can be expressed as:

$u_{i}\left(x-x_{i}\right)+v_{i}\left(y-y_{i}\right)=0$

Since the line $u_{i}\left(x_{i}^{\prime}, x_{i}\right)+v_{i}\left(y_{i}^{\prime}, y_{i}\right)=0$ is theoretically valid, we have:

$\chi^{2}(t)=\sum_{i=1}^{n}\left[u_{i}\left(x_{i}^{\prime}(t)-x_{i}\right)+v_{i}\left(y_{i}^{\prime}(t)-y_{i}\right)\right]^{2}$

$t_{\min }=t^{\prime}+D^{-1} \bullet\left[-\nabla \chi^{2}\left(t^{\prime}\right)\right]$

where $t^{\prime}$ is the approximate value of matching parameters; D is the Hessian matrix; $\nabla x^{2}$ is the gradient matrix of $x^{2}$. When $x^{2}(t) \rightarrow \min$, the matching positions should be adjusted to enhance the accuracy. In general, the stable solution of the new positions can be obtained after three iterations at $t_{\text {min }}$.

\section{ADAPTIVE TEMPLATE MATCHING DETECTION}

The detection of BGA solder joints involves ROI selection, template generation and template match searching. The work flow is illustrated in Figure 4. Below is a detailed introduction to the detections steps.

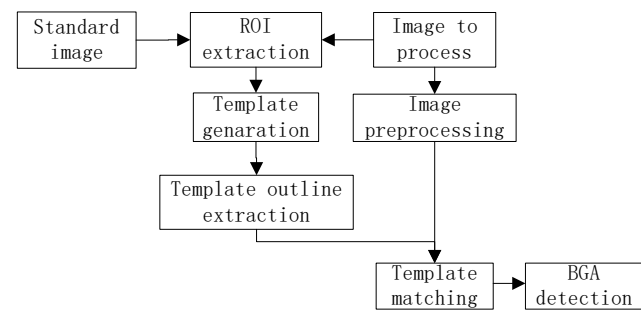

Figure 4. Workflow of BGA solder joint detection

The ROI should be selected from the standard image and then separated from the original image to serve as a template. The sizes of BGA solder joints can be identified with an Xray image of any magnification factor. As mentioned before, the BGA solder joints are not strictly spherical and differ slightly from each other in shape. Thus, it is possible to obtain the ROI by choosing the proper solder joints from the original image, and thus generate the target template suitable for image matching with deformations in $\mathrm{X}$ and $\mathrm{Y}$ directions.

The ROI is a single-channel greyscale image. Since the relative minimum can be extracted from its relative greyscale histogram, the histogram should be smoothened by Gaussian filtering to reduce the number of relative minimums. These values should be taken as the initial parameters of thresholding. With a continuously expanding Gaussian smoothing window, the iteration should not be terminated until the only minimum is extracted from the histogram. In this way, the edge information can be extracted from the selected ROI, forming an effective template.
Next, it is necessary to create a shape model based on the extracted edge information. Based on the shape, the final edge template should be established with the proper layers of the image pyramid and rational range of greyscale. The edge shape helps to locate the positions in the target image that are consistent with the edge template. Meanwhile, the normalized coordinates will be returned at the sub-pixel level of accuracy. Scaling and rotation of the template may occur during the matching process, aiming to match the edge shape of the target images. The workflow of BGA edge template extraction is depicted in Figure 5.

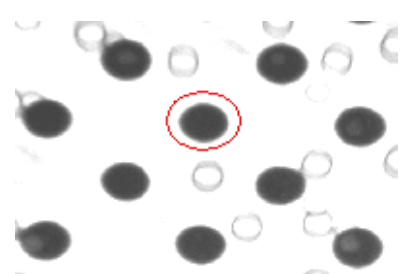

(a)ROI selection

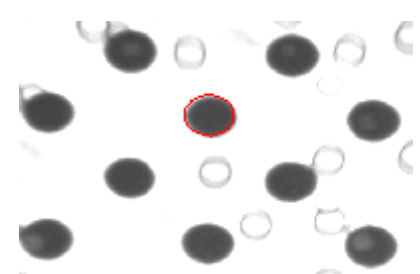

(b)Solder joint extraction
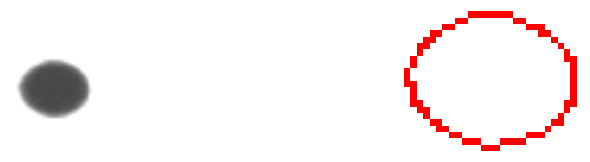

(c)Extracted solder joints

(d)Edge template

Figure 5. Workflow of BGA edge template extraction

\section{TEST RESULTS AND ANALYSIS}

The proposed adaptive template matching method was applied to the detection of BGA solder joints in a target image. The detection test was conducted under the voltage of $90 \mathrm{kV}$ and the current of $70 \mu \mathrm{A}$. The diameter of each solder joint is about 150 pixels in the image. Sequential detection was performed on BGA solder joints of different PCBs. According to the detection results in Figure 6 and Table 1, the detection rate fluctuated first and then stabilized with the continued expansion of the sample size.

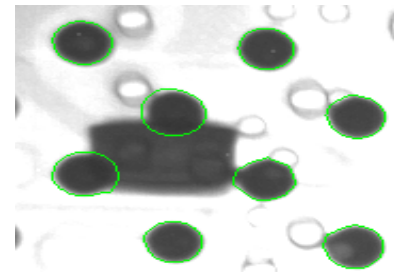

(a) Partial coverage

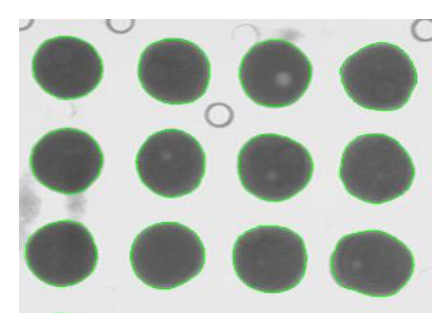

(c) Irregular deformation

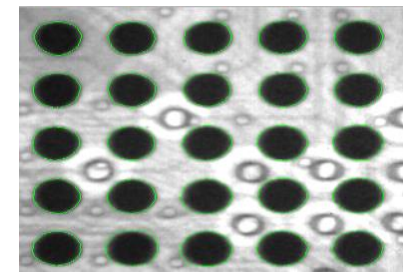

(b)Nonuniform brightness

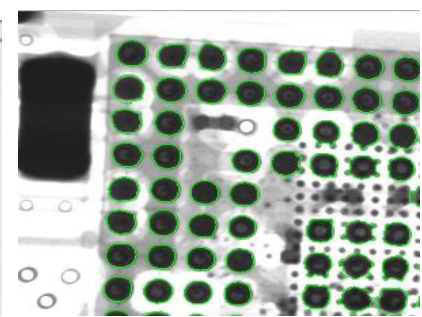

(d) Complicated background
Figure 6. Results of adaptive template matching detection 
Table 1. Results of BGA solder joint detection

\begin{tabular}{ccccc}
\hline Total No. & Missed No. & False No. & (a) $(\%)$ & (b) $(\%)$ \\
\hline 20 & 1 & 0 & 95.0 & 0 \\
\hline 43 & 3 & 0 & 93.0 & 0 \\
\hline 79 & 4 & 0 & 94.9 & 0 \\
\hline 104 & 4 & 1 & 96.2 & 0.96 \\
\hline 156 & 7 & 2 & 95.5 & 1.28 \\
\hline 217 & 8 & 2 & 96.3 & 0.92 \\
\hline
\end{tabular}

Notes: 1. (a)Detection rate of BGA solder joints, (b)False detection rate of BGA solder joints. 2.Detection rate is the quotient between the number of detected solder joints and the total number of solder joints; False detection rate is the quotient between the incorrectly detected solder joints and the total number of solder joints.

In Figure 6(a), the edges of the solder joints are partially covered. The edges of the covered part were predicted by the adaptive template matching method based on the detected edge profile, forming a complete profile of BGA solder joints. In Figure 6(b), the background brightness and the greyscale change from place to place. By the adaptive template matching, the background noises were reduced and the edge features of solder joints were enhanced through image smoothing and the detection of edge information. Thus, the matching process is not sensitive to the greyscale gradient in the local area. In Figure 6(c), the edges of the BGA solder joints suffer from irregular deformations. During the adaptive template matching, the edge features were zoomed in or out and the template underwent angular rotation before match searching, and the target solder joints were obtained according to the final similarity scores. In this way, the recognition rate was enhanced for BGA solder joints with similar key features and slight local differences. In Figure 6(d), the target image has a complicated background with a variety of noises. It can be seen that the proposed method still achieves a good detection results.

In summary, the proposed adaptive template matching method realized high detection accuracy when BGA solder joints have partial coverage, nonuniform brightness, irregular deformation and complicated background. Whichever the condition, our method can always locate the solder joints rapidly and precisely against the edge template. Suffice it to say that the adaptive template matching method is robust and accurate for solder joint detection under various conditions.

\section{CONCLUSION}

This paper puts forward a novel BGA solder joint detection method based on adaptive template matching. The new method takes the edge shape of BGA solder joints as the template, and accelerates the matching speed using image pyramid and similarity threshold. Besides, the deformation was calculated according to the returned edge direction vectors, and the matching accuracy was enhanced through position adjustment and transformation. Experimental results prove that the proposed method can detect BGA solder joints efficiently and accurately at any conditions. The research findings shed new light on the industrial application of BGA solder joint detection.

\section{ACKNOWLEDGMENT}

This paper is made possible thanks to the generous support from the Natural Science Basic Research Program of Shaanxi
Province, China (Grant No.:2017ZDJC-23; 2017JQ5014).

\section{REFERENCES}

[1] Mak CW, Afzulpurkar NV, Dailey MN, Saram PB, Bayesian A. (2014). Approach to Automated Optical Inspection for Solder Jet Ball Joint Defects in the Head Gimbal Assembly Process. IEEE Transactions on Automation Science and Engineering 11(4): 1155-1162. https://doi.org/ 10.1109/TASE.2014.2305654

[2] Said AF, Bennett B, Karam L. (2010). Robust automatic void detection in solder balls, Acoustics Speech and Signal Processing (ICASSP). 2010 IEEE International Conference, Dallas, 1650-1653. https://doi.org/ 10.1109/ICASSP.2010.5495524

[3] Ieamsaard J, Yammen S, Muneesawang P. (2015). Vertical Edge Detection Based Automatic Optical Inspection for Solder Jet Ball Joint Defect on Head Gimbal Assembly, 24-27. https://doi.org/ 10.1109/ECTICon.2015.7206989

[4] Shao HP, Hyum D. (2011). BGA void detection in Xray images based on a new blobdetector, Image and Signal Processing (CISP). 2011 4th International Congress, Shanghai, 1847-1850. https://doi.org/ 10.1109/CISP.2011.6100555

[5] Du X. (2016). A mechanical parts inspection model based on image classification technology. Academic Journal of Manufacturing Engineering 14(3): 14-21.

[6] Saenthon A, Kaitwanidvilai S. (2010). Development of new edge detection filter based on genetic algorithm: An application to a soldering joint inspection. International Journal of Advanced Manufacturing Technology 46: 1009-1019. https://doi.org/ 10.1007/s00170-009-2157-x

[7] Zhang HZ, Wang XL, Li Y, Liu YJ, Zhou D, Hou BH. (2017). A new image identification method for elevator door protection. Academic Journal of Manufacturing Engineering 15(2): 15-22.

[8] Nss M, Pkdv Y, Fookes C. (2011). Design and development of automatic visual inspection system for PCB manufacturing. Robotics and Computer Integrated Manufacturing, 949-962. https://doi.org/ 10.1016/j.rcim.2011.03.007

[9] Dong Y, Wu CS, Lv QH, Li HK, Guo HM. (2015). Study of CO2 fluid density calculation model based on grayscale image. International Journal of Heat and Technology 33(1): 161-166. https://doi.org/ 10.18280/ijht.330122

[10] Abdelhameed MM, Mechatron ED. (2014). A robust methodology for solder joints extraction. Computer Engineering \& Systems (ICCES), 268-273. https://doi.org/ 10.1109/ICCES.2013.6707217

[11] Rafiee SE, Sadeghiazad MM. (2015). 3D numerical analysis on the effect of rounding off edge radius on thermal separation inside a vortex tube. International Journal of Heat and Technology 33(1): 83-90. https://doi.org/ 10.18280/ijht.330112

[12] Bohm J, Wolter KJ, Heuer H. (2014). Solder Joint Inspection with Induction Thermography. 2014 IEEE 64th Electronic Components and Technology Conference, 1509-1516. https://doi.org/ 10.1109/ECTC.2014.6897494 
[13] Hasnine M, Univ A. (2014). Exploration of aging induced evolution of solder joints using nanoindentation and microdiffraction. 2014 IEEE 64th Electronic Components and Technology Conference (ECTC), 379394. https://doi.org/ 10.1109/ECTC.2014.6897315

[14] Said AF, Bennett BL, Toth F, Karam LJ. (2010). Nonwet solder joint detection in processorsockets and BGA assemblies. 2010 Proceedings 60th Electronic Components and Technology Conference (ECTC), 1147-1153. https://doi.org/ 10.1109/ ECTC.2010.5490846

[15] Pierce BL, Shelton DJ, Longbotham HG, Baddipudi S. (1994). Automated inspection of through hole solder joints utilizing X-ray imaging. IEEE Aerospace and Electronic Systems Magazine, 28-32. https://doi.org/ 10.1109/62.260040

[16] Park JS, Tou JT. (1990). A solder joint inspection system for automated printed circuit board manufacturing. 1990 IEEE IntConf Rob Autom, 12901295. https://doi.org/ 10.1109/ROBOT. 1990.126177

[17] Koh KC, Choi HJ, Kim JS, Cho HS. (2001). A statistical learning-based object recognition algorithm for solder joint inspection. Proceedings of SPIE-The International Society for Optical Engineering, 260-267. https://doi.org/ 10.1117/12.444096

[18] Lahari MS, Hijer R, Khuwaja GA. (2011). Efficient techniques for BGA solder joint identification in low resolution X-ray images. 2011 IEEE GCC Conference and Exhibition, 128-131. https://doi.org/ 10.1109/IEEEGCC.2011.5752465
[19] Deokhwa H, Heechan P, Hyungsuck C. (2009). Design of a multi-screen deflectometer for shape measurement of solder joints on a PCB. IEEE International Symposium on Industrial Electronics, 127-132. https://doi.org/ 10.1109/ISIE.2009.5213206

\section{NOMENCLATURE}

T dimensionless template function

s dimensionless subgraph

$\mathrm{R}$ dimensionless similarity of template $\mathrm{T}$ and subgraph

$\mathrm{p}$ dimensionless point on the template edge

d dimensionless direction vector

M dimensionless second order standard rotation matrix

q dimensionless match of template with original image

t' dimensionless approximate matching parameter values

D dimensionless the Hessian matrix

\section{Greek symbols}

$\nabla \chi^{2} \quad$ the gradient matrix

\section{Subscripts}

i horizontal coordinate of the pixel

$\mathrm{j} \quad$ vertical coordinate of the pixel

$\mathrm{x}$ horizontal coordinate of edge point

$\mathrm{y}$ vertical coordinate of edge point 